PART 3

SOLAR FLARES 


\title{
ULTRAVIOLET EMISSION LINE PROFILES OF FLARES AND ACTIVE REGIONS
}

\author{
G. E. BRUECKNER \\ E. O. Hulburt Center for Space Research, Naval Research Laboratory, \\ Washington, D.C. 20375, U.S.A.
}

\begin{abstract}
A preliminary description of ultraviolet spectra of active regions and flares, photographed from Skylab by the Naval Research Laboratory's UV Spectrograph is given. The findings can be summarized as follows: (1) Line profiles of medium ionized lines (transition zone lines) show the most pronounced broadenings and shifts in flares and flare like events. (2) Typical full width at half maximum of these lines correspond to Doppler-velocities of $70 \mathrm{~km} \mathrm{~s}^{-1}$. (3) Shifts of the same magnitude can be observed. (4) Intersystem lines are not broadened nor shifted. (5) Forbidden coronal lines and intersystem lines become enhanced in the flare spectrum at the moment, when the turbulence seen in the allowed transitions, disappears. (6) A very broad line at $1354.2 \AA$ which appears only in flare spectra, seems to be the forbidden transition ${ }^{3} P_{1}-{ }^{3} P_{0}$ of Fe xxI.
\end{abstract}

The instrument covered a spectral range from $970 \AA$ to $4000 \AA$, but in the following we shall concentrate on the wavelength range $1170 \AA$ to $1950 \AA$, where all the important emission lines are located.

There is no real imaging along the 1' long slit of the spectrograph, which is only pseudo-stigmatic. A source with even intensity distribution over the whole slit produces a smooth spectrum in the spectrograph's focal plane. But a point source, illuminating the slit only at one point, would produce a different intensity distribution perpendicular to the dispersion in the spectrum. The spectrum would show a distinct peak with long wings measured perpendicular to the dispersion because of some residual imaging capabilities which are inherited in a pseudo-stigmatic imaging system. It seems therefore one can conclude, that spatial structure along the slit exists if one finds an uneven, structured spectrum perpendicular to the dispersion. And vice versa, if the spectrum has a smooth appearance one can conclude that the illuminating source has a fairly even intensity distribution over the 1' long slit. It is almost impossible to quote numbers. But the experience with the instrument shows, that only very small structures having very high contrast with their surroundings can be distinguished in the focal plane of the pseudo-stigmatic instrument. One has to keep these imaging capabilities of the spectrograph in mind when interpretations of the spectra are made.

A spectral resolution of approximately $0.07 \AA$ could be obtained, including all contributing factors to the instrument profile from the slit width, film and microphotometer effects. This resolution is sufficient, to derive emission line profiles of chromospheric, transition zone and coronal lines.

Figure 1 shows two spectra of the 1973, June 15 flare. The upper spectrum was photographed approximately $1 \mathrm{~min}$ after the $\mathrm{X}$-ray peak during the maximum phase of the flare, the lower spectrum for comparison 26 min later when the flare had ceased. Two different exposure times have been selected in order to show spectra of approxi- 


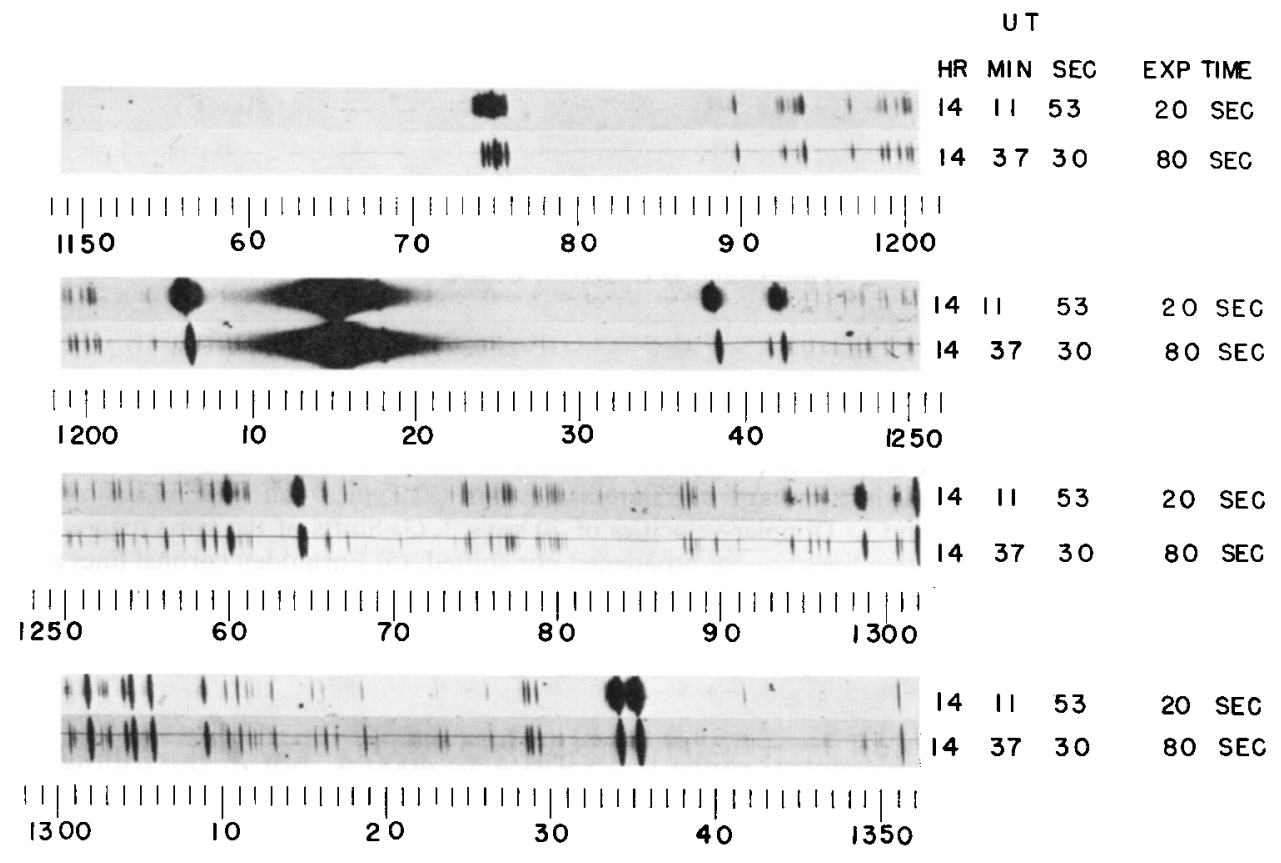

Fig. 1. Spectra of the 1973 , June 15 th flare, $1150 \AA$ to $1350 \AA$. Upper spectrum: Flare during maximum phase. Lower spectrum: Same pointing, after flare has ceased.

mately the same photographic density. (The spectra as photographed from Skylab consist always of a series of four different exposures to bridge a wide dynamic range from the faintest to the strongest lines.)

At $1175 \AA$ we see the $C$ III multiplet resolved. It is strongly enhanced in the flare and all lines are broadened.

At $1204 \AA$ we observe the weak intersystem line from S v. This line should be density sensitive if the local density exceeds a certain value. It should be noted that this line, like all other intersystem lines does not show any broadening in the flare spectrum. We may therefore conclude that it does not originate in the flare plasma itself. The intersystem lines seem to come from the surrounding area, where they are enhanced like all other lines.

$\mathrm{L} \alpha$ is overexposed, but it is enhanced approximately $3 \times$ in the flare.

The $\mathrm{O} v{ }^{1} S-{ }^{3} \mathrm{P}$ intersystem line at $1218 \AA$ behaves similar like the $\mathrm{S} v$ line. No broadening of the line profile nor any enhancement during the flare phase can be observed.

At $1240 \AA$ follows the $\mathrm{N} v$ resonance doublet, which is enhanced and broadened in the flare spectrum. Both components are blue shifted contrary to the Si III resonance line at $1206 \AA$ which is reshifted during this phase of the flare. The Fe xII forbidden line at $1241.7 \AA$ is not enhanced in the flare spectrum during the early phase. 


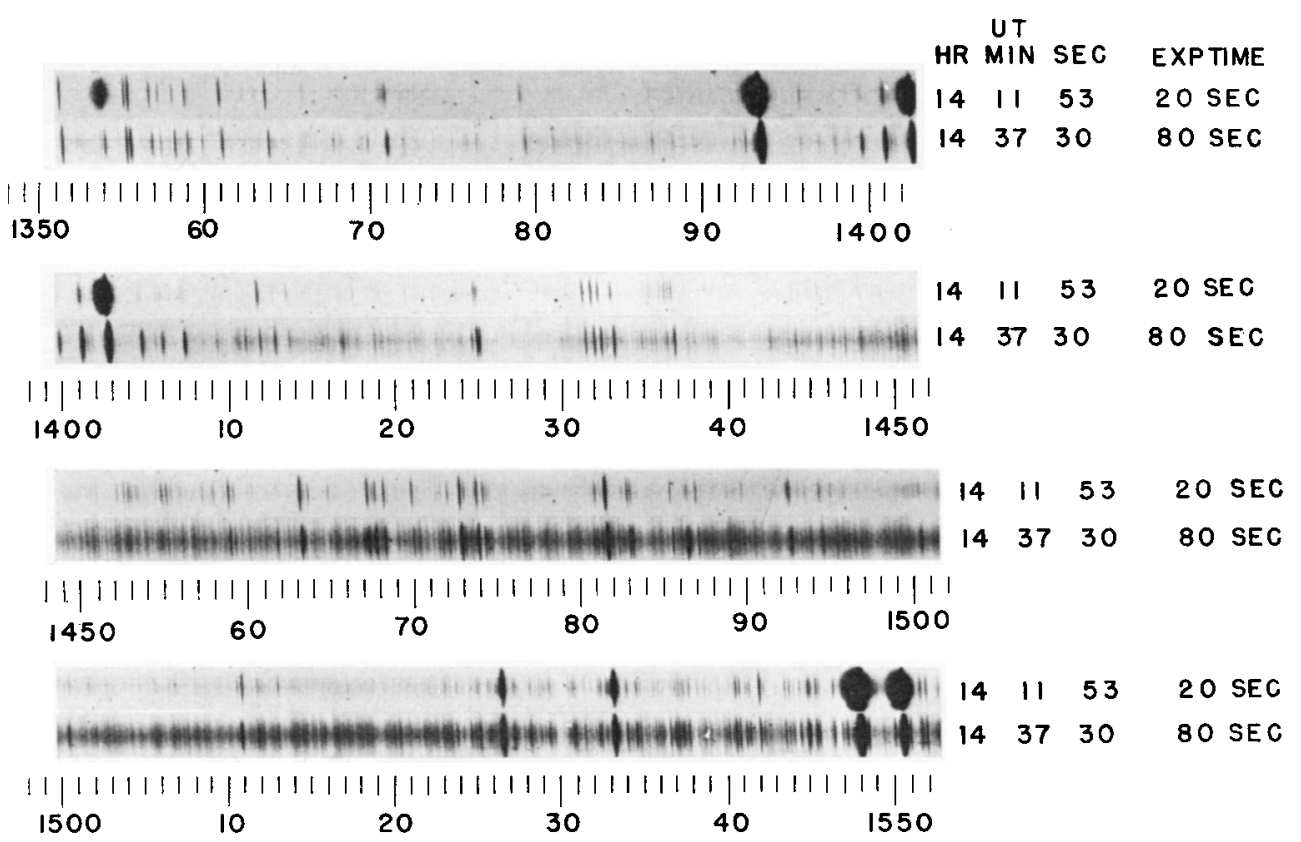

Fig. 2. Spectra of the 1973 , June 15 th flare, $1350 \AA$ to $1550 \AA$. Upper spectrum: Flare during maximum phase. Lower spectrum: Same pointing, after flare has ceased.

The Si II doublet at $1260 \AA$ and $1265 \AA$ shows less broadening in the flare spectrum than the Si III line at $1206 \AA$.

$\mathrm{O}_{\mathrm{I}}$ at $1300 \AA$ is enhanced, but its profile is only slightly changed. Weak lines near the oxygen triplet which show flare broadening are from Si II and Si III.

$C_{\text {II }}$ at 1334 and $1335 \AA$ shows a blue shifted flare profile. At 1349 we find the forbidden Fe XII line, which is not enhanced in the flare spectrum during the early phase. An unidentified line of similar behavior can be seen at $1323.8 \AA$. Another unidentified sharp line at $1342 \AA$ shows a very strong enhancement.

The most peculiar line in flare spectra can be found at $1354 \AA$ (Figure 2). This line becomes very bright in flares, it is very broad and has no counter part in active regions. Feldman et al. (1974) have calculated the wavelength of the forbidden transition ${ }^{3} P_{1}-{ }^{3} P_{0}$ of Fe XXI in the carbon sequence to be $1355 \AA \pm 3 \AA$. An ion temperature of approximately $20 \times 10^{6} \mathrm{~K}$ can be derived from the halfwidth of this line. The intensity change of this line with time is distinctly different from the intensity change of the transition zone lines. We also do not see any structure of the line profile along the slit. All these observations indicate that the 1354 line indeed comes from a region of the flare, where different physical conditions prevail than those in the area, where the transition zone lines are formed. It is therefore very likely that the identification will hold.

At 1355.6 and 1358.5 the $\mathrm{O}$ I intersystem doublet ${ }^{3} P-{ }^{5} S$ does show only a very slight enhancement in the flare spectrum. Because all other neutral lines from allowed 

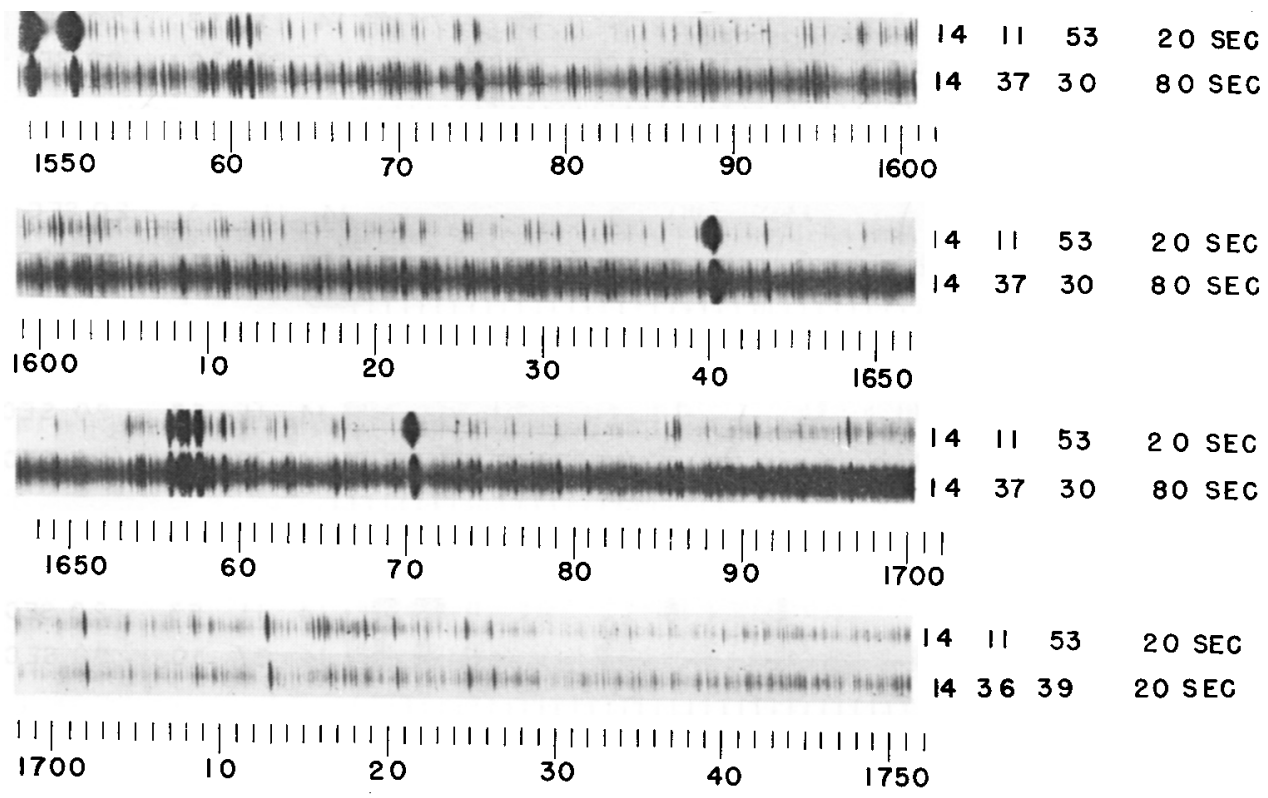

Fig. 3. Spectra of the 1973 , June 15 th flare, $1550 \AA$ to $1750 \AA$. Upper spectrum: Flare during maximum phase. Lower spectrum: Same pointing, after flare has ceased.

\begin{tabular}{|c|c|c|c|}
\hline $\mathrm{HR}$ & $\begin{array}{r}\text { UT } \\
\text { IR MIN }\end{array}$ & SEC & EXP TIME \\
\hline 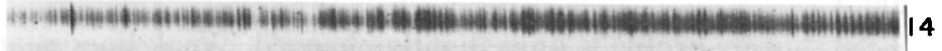 & 411 & 53 & 20 SEC \\
\hline 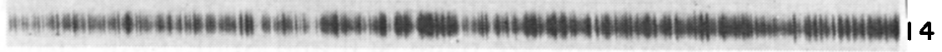 & 36 & 39 & $20 \mathrm{SEC}$ \\
\hline $11111111111|111111111| 111111111|111111111| 111111111111$ & & & \\
\hline $\begin{array}{ll}70 & 80\end{array}$ & & & \\
\hline
\end{tabular}

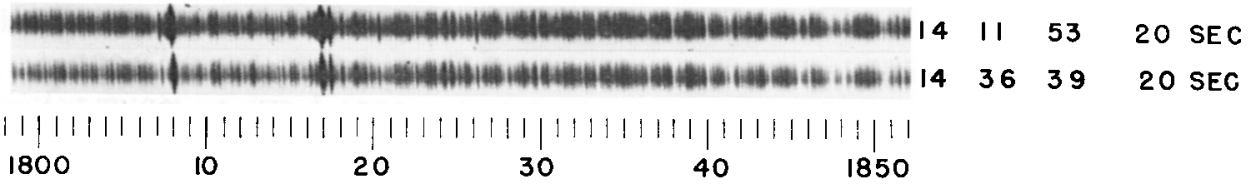

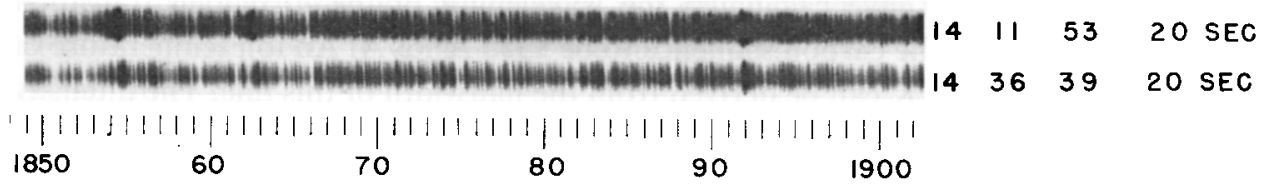

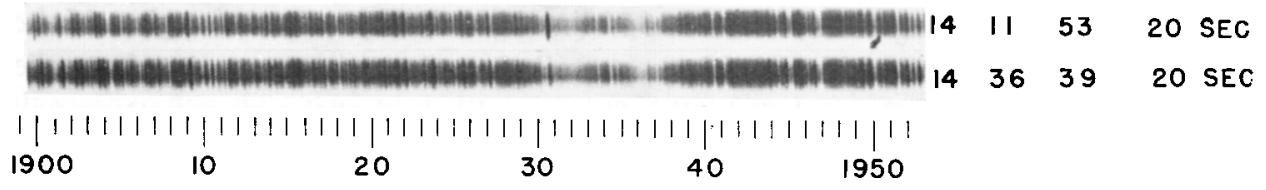

Fig. 4. Spectra of the 1973 , June 15 th flare, $1750 \AA$ to $1950 \AA$. Upper spectrum: Flare during maximum phase. Lower spectrum: Same pointing, after flare has ceased. 


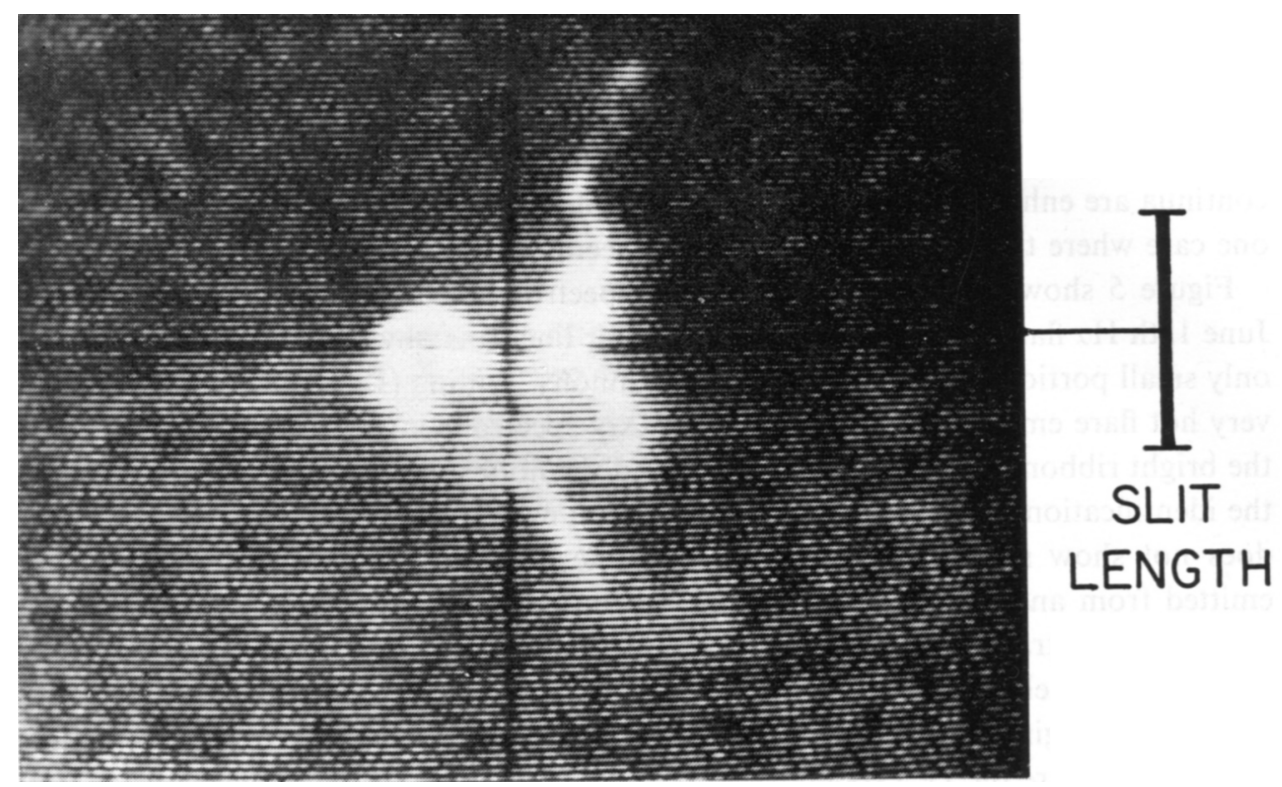

\section{FLARE, JUNE $15^{\text {th }} 1973$}

Fig. 5. Ha image of the June 15th flare, transmitted from the display on board Skylab. The spectrograph slit was positioned along the vertical crosshair.

transitions in this region of the spectrum show a stronger enhancement, we can conclude that in the chromosphere where $\mathrm{O} I$ is formed, the collision rate during a flare is enhanced.

In the flare spectrum the most broadened lines are the Si IV lines at $1400 \AA$. Also around $1400 \AA$ we find the ${ }^{2} P-{ }^{4} P$ intersystem lines of $\mathrm{O}$ IV, which are not enhanced and not broadened in the flare spectrum. The same applies to the $S$ IV ${ }^{2} P-{ }^{4} P$ intersystem lines at 1406 and $1416.9 \AA$.

The Si II doublet at 1526 and $1533 \AA$ does not show significant broadening in the flare, contrary to the Si II doublet at $1260 \AA$.

The C IV resonance doublet at $1540 \AA$ shows a very broad line profile and strong enhancement in the flare spectrum.

$\mathrm{H} \alpha$ of $\mathrm{He}$ II (Figure 3) at $1640 \AA$ seemed to be broadened, but the line is blended with an $\mathrm{Fe}$ II line, therefore it is difficult to determine the contribution of $\mathrm{He}$ and $\mathrm{Fe}$ to the composite line profile.

The same applies to $\mathrm{Al}$ II at $1670 \AA$. Fe II does not show signs of significant broadening, which can be seen from the appearance of the many isolated Fe II lines in this region of the spectrum.

The $\mathrm{N}_{\text {III }}$ intersystem line at $1754 \AA$ is slightly weakened in the flare spectrum (Figure 4). This is not the case for the Si III intersystem line at $1892 \AA$ which does not show any intensity change or broadening. 
Finally we are pointing out that the continuum below $1520 \AA$, (Si triplet continuum) does show only a very slight enhancement in the flare spectrum, while we cannot see any change in the continuum above $1520 \AA$. Also, the Al autoionization lines at $1934 \AA$ do not change. This is not the case in all spectra of flares. There are spectra where both continua are enhanced, particularly the Si triplet continuum. We have found at least one case where the Al-autoionization lines seem to disappear.

Figure 5 shows the slit position of the spectrograph as it was pointed onto the June 15 th $\mathrm{H} \alpha$ flare along the vertical cross hair line. It is obvious that the slit covered only small portions of the very bright $\mathrm{H} \alpha$ ribbons. Widing (1975) has found, that the very hot flare emission, as seen in the Fe XxIV spectroheliograms, is located between the bright ribbons. Therefore the slit covered the hottest area of the flare. This makes the identification of the $1354 \AA$ line with Fe XXI even more likely. The line at $1354 \AA$ does not show structure perpendicular to the dispersion, which means, that it is emitted from an area comparable with the slit area, contrary to the appearance of the broadened transition zone lines. Widing measured the size of the Fe XXIV emission in the spectroheliograms to be approx. 30". It is therefore safe to assume, that the $\mathrm{Fe}$ XXI line originates from the same hot coronal plasma above the explosive region of the flare where the Fe XXIV emission comes from. While a time sequence of spectra was taken the pointing had not been changed.

We shall now discuss the time dependence of the spectra. Figure 6 shows a series of spectra covering a time interval of $25 \mathrm{~min}$, taken all with $20 \mathrm{~s}$ exposure time. The turbulent phase of the flare, which is distinguished by the very broad line profiles of the medium ionized lines like Si II at $1260 \AA$, Si III at $1206 \AA$ and N V at $1240 \AA$ lasts approximately $7 \mathrm{~min}$. During this phase, the broadening gradually decreases in all the lines mentioned. But we also see changing shifts from predominantly blue shifts at the beginning to red shifts during the later turbulent phase. We also note, that the Si III line at 1206 exhibits a red shift at the same time when Si II at 1260 is blue shifted. (Upper two spectra in Figure 6.) After the turbulent phase has ceased at $14 \mathrm{~h}, 22 \mathrm{~min}$, $26 \mathrm{~s}$ abruptly the forbidden Fe XII lines at $1240 \AA$ appears very strong and then decreases gradually toward the end of the sequence. While intensity and line width of the $\mathrm{C}$ II lines at $1335 \AA$ (Figure 7) are decreasing with time, beginning with the first spectrum, the Fe XXI $1354 \AA$ line increases in intensity during the first $3 \mathrm{~min}$, to disappear gradually toward the end of the sequence. $C$ II behaves very similar like all other transition zone lines. $1354 \AA$ should therefore not originate in the transition zone. It also cannot be attributed to the chromosphere, because of its disappearance after the flare has ceased and its very broad line profile.

The same behavior of the medium ionized lines can be seen in Figure 8, which shows the broadening of Si IV. At $14 \mathrm{hr}, 22 \mathrm{~min}, 26 \mathrm{~s}$ when the Si IV line broadening stops abruptly, the intersystem lines of O IV and S IV become very intense.

Figure 9 demonstrates another example of the selective appearances of the line shifts in different ions. While C IV at $1550 \AA$ shows a significant red shift, at $14 \mathrm{hr}$, $18 \mathrm{~min}, 23 \mathrm{sec}$, the $\mathrm{Si}$ II doublet remains unshifted. The slight enhancement of the $\mathrm{Si}$ triplet continuum below $1520 \AA$ lasts very long through $14 \mathrm{~h}, 28 \mathrm{~min}$. Another notice- 


$$
\begin{aligned}
& \text { 岀 }
\end{aligned}
$$

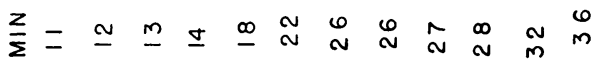

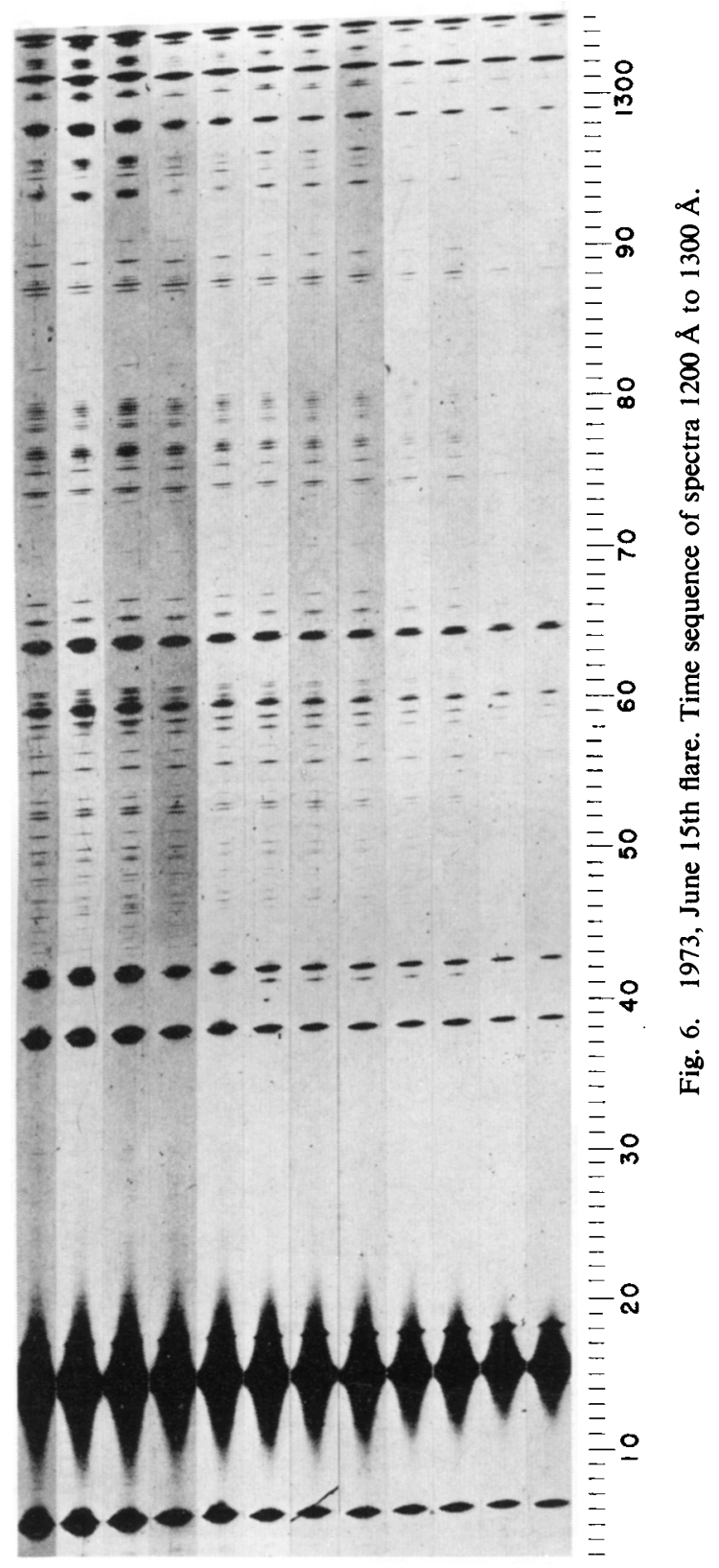




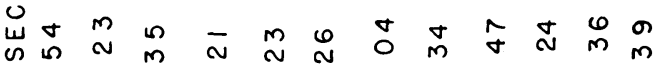

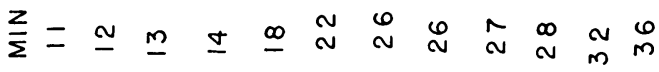

$$
\begin{aligned}
& \stackrel{\square}{I}
\end{aligned}
$$

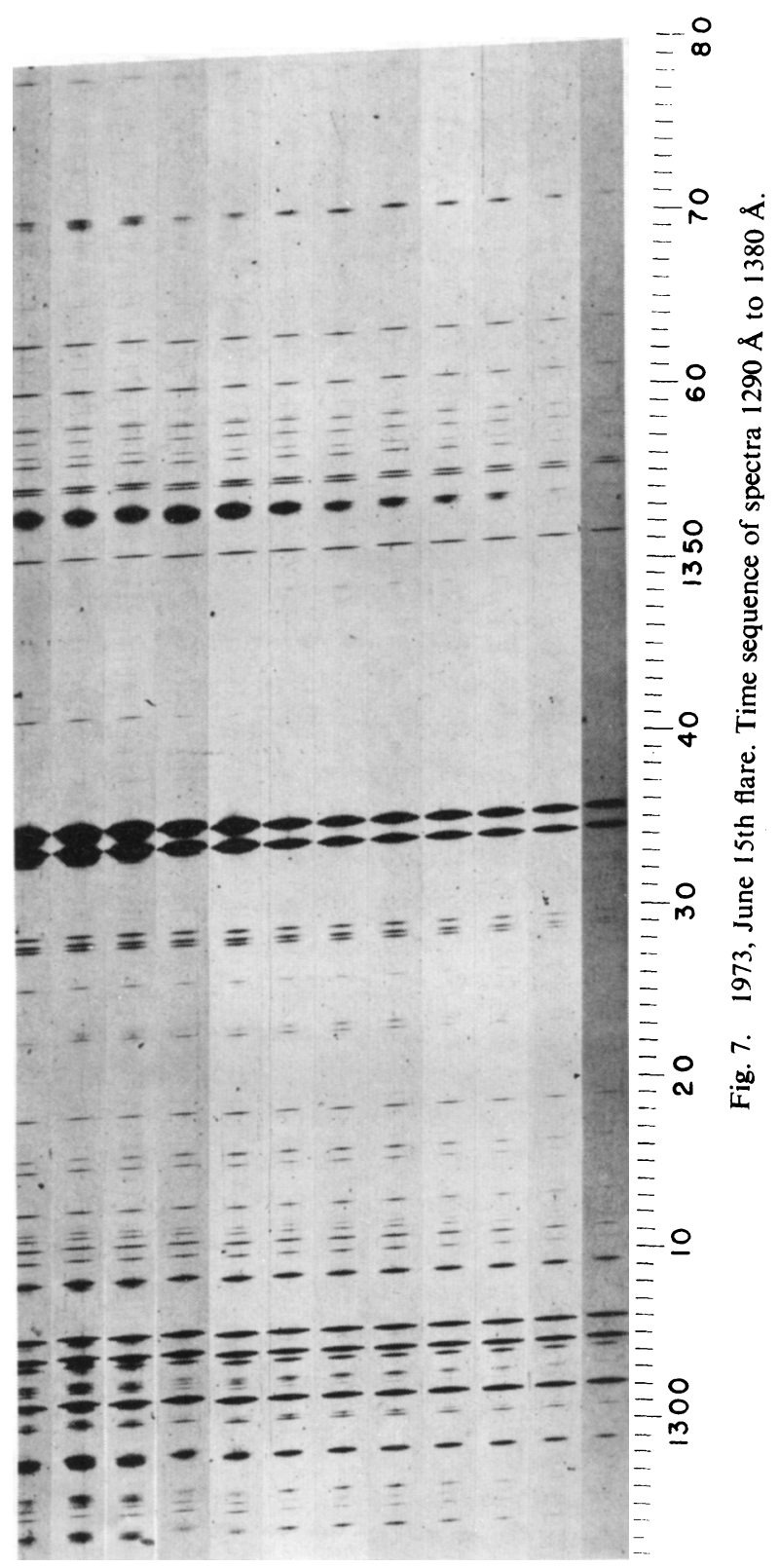




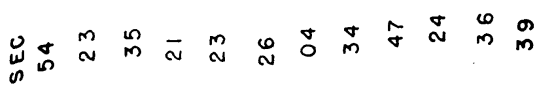

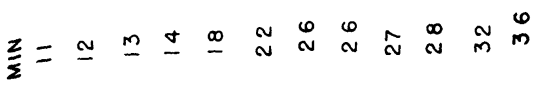

$\stackrel{a}{I}$

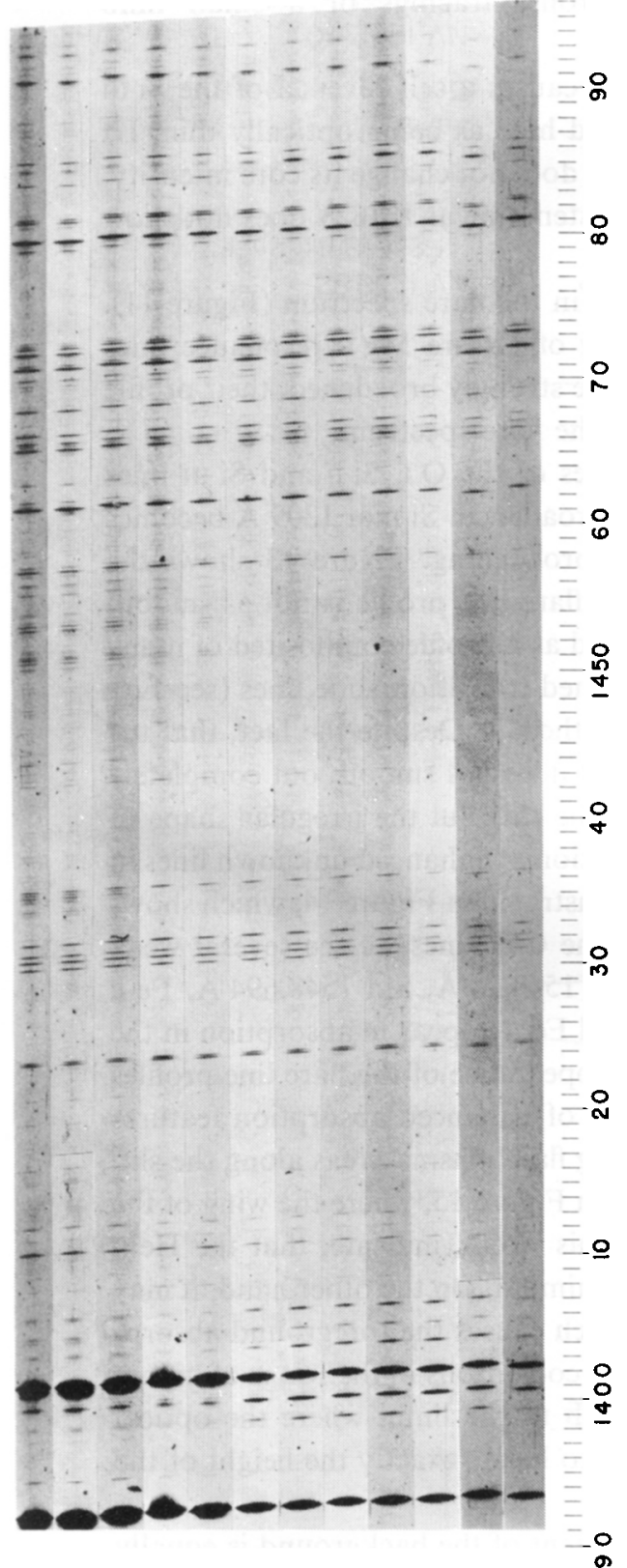

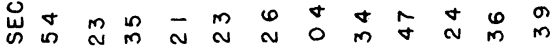

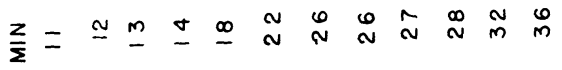

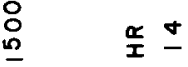

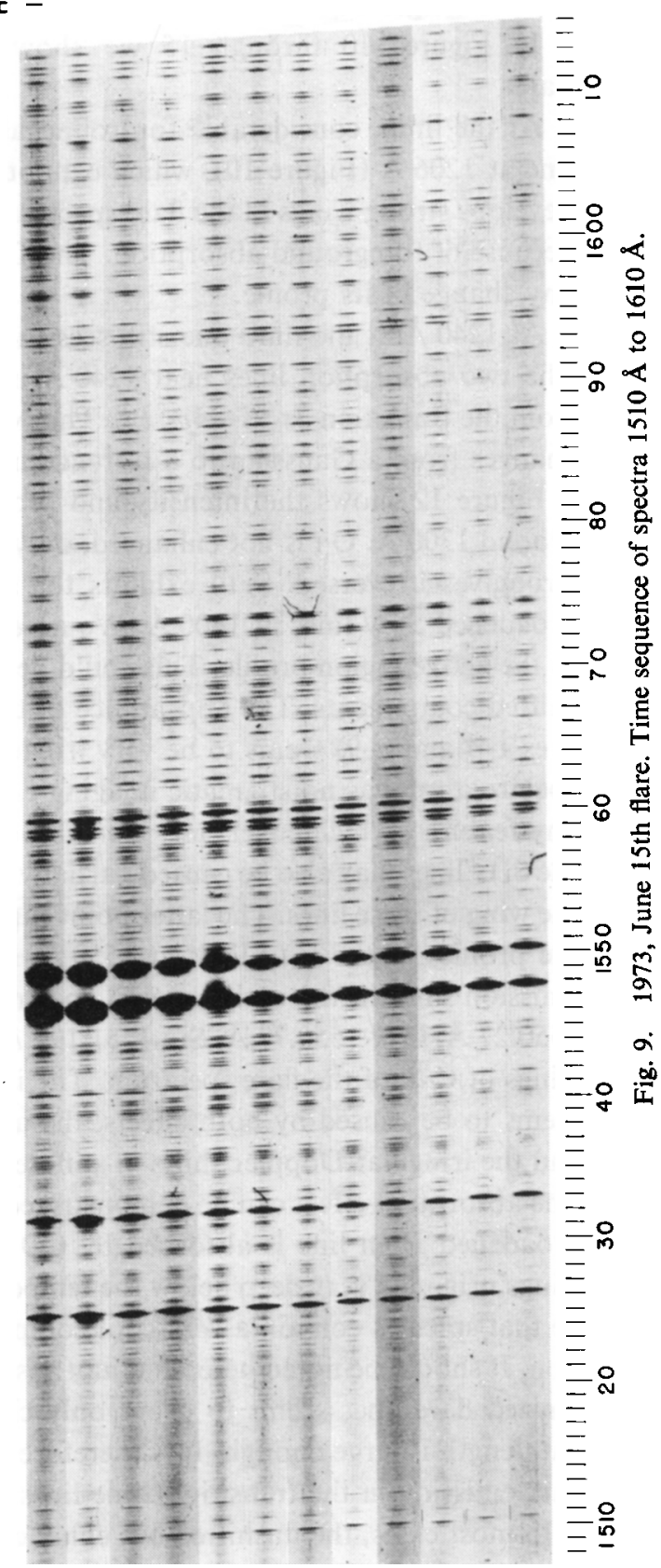


able effect can be demonstrated in this portion of the spectrum. At the beginning of the flare all neutral and $1 \times$ ionized lines are strongly enhanced in the short wavelength part of the spectrum around $L \alpha$. This is not the case for the lines of the same origin at longer wavelength. It should be noted that there is no continuum at shorter wavelength, while above $1520 \AA$ the $\mathrm{Si}$ singlet and triplet continuum becomes stronger toward longer wavelength.

In Figures 10 through 15 we show some profile tracings of a small limb flare.

At the limb, considerable forground absorption causes a self reversal of the Si III line at $1206 \AA$ (Figure 10), which cannot be treated here as being optically thin. $\mathrm{L} \alpha$ develops strong wings in the flare spectrum, while it does not change its core intensity, because of foreground absorption. The $\mathrm{O} v$ intersystem line at $1218 \AA$ does not show any change of its profile.

At $1240 \AA$ at the limb one still sees the $\mathrm{L} \alpha$ wing in the flare spectrum (Figure 11). The two absorption lines near $1240 \AA$ in the wing of $\mathrm{L} \alpha$ are $\mathrm{Mg}$ II resonance lines from the transition $3 s^{2} S-4 p^{2} P^{0}$. The $\mathrm{N} v$ lines are strongly broadened, their profile changes from a Gaussian to a Lorentzian type in the flare spectrum.

Figure 12 shows the intensity and profile changes of the $\mathrm{O}$ I, Si II and $\mathrm{Si}$ III lines around $1300 \AA$. O I is not enhanced, only slightly broadened. Si II at $1309 \AA$ becomes strongly selfreversed. Si III exhibits the strongest broadening. Figure 13 shows the broadened Si Iv lines at $1400 \AA$. We note, that the flare line profile is not as smooth as the active region profile. This could be interpreted as a profile composited of many shifted components. The appearance of the broadened transition zone lines (see Figures 1-4 and 6-9) seems to be very irregular along the slit. Despite the fact, that the spectrograph has no stigmatic imaging capability, it does not smooth out completely any features, which cover only a small portion of the slit. But the irregular shape of the Si IV lines may also be caused partially by absorption of enhanced unknown lines in the wing of these lines. The latter point can be demonstrated in Figure 14, which shows the profiles of the $C_{\text {IV }}$ lines at $1550 \AA$. Between the $C_{\text {IV }}$ lines we see several weak emission lines in the active region spectrum. (C IV $1548.20 \AA$, Si I $1548.694 \AA$, Fe II 1550.27 $\AA$, C IV $1550.77 \AA$, Si I $1551.199 \AA$.) Si I and Fe II appear in absorption in the wings of $\mathrm{C}$ IV of the flare spectrum. The irregular appearance of the flare line profiles seems to be caused by both effects, the appearance of enhanced absorption features and the irregular Doppler shifts of different emitting flare plasma areas along the slit. The absorption effect can be demonstrated further in Figure 15, where the wing of the broadened $\mathrm{He}$ II line is absorbed by $\mathrm{CO}$ bands. This would indicate, that the $\mathrm{He}$ II wings originate very deep below the temperature minimum. On the other hand, it may be that spicules contain a very cool component which causes the foreground absorption. It should be noted here, that any absorption of cooler ions in the wings of higher ionized flare lines seems to occur only close enough to the limb, where the optical pathlength is large enough. It is therefore not easy, to locate exactly the height of the flare emission in the transition zone lines.

In most cases, the unshifted but enhanced component of the background is equally 

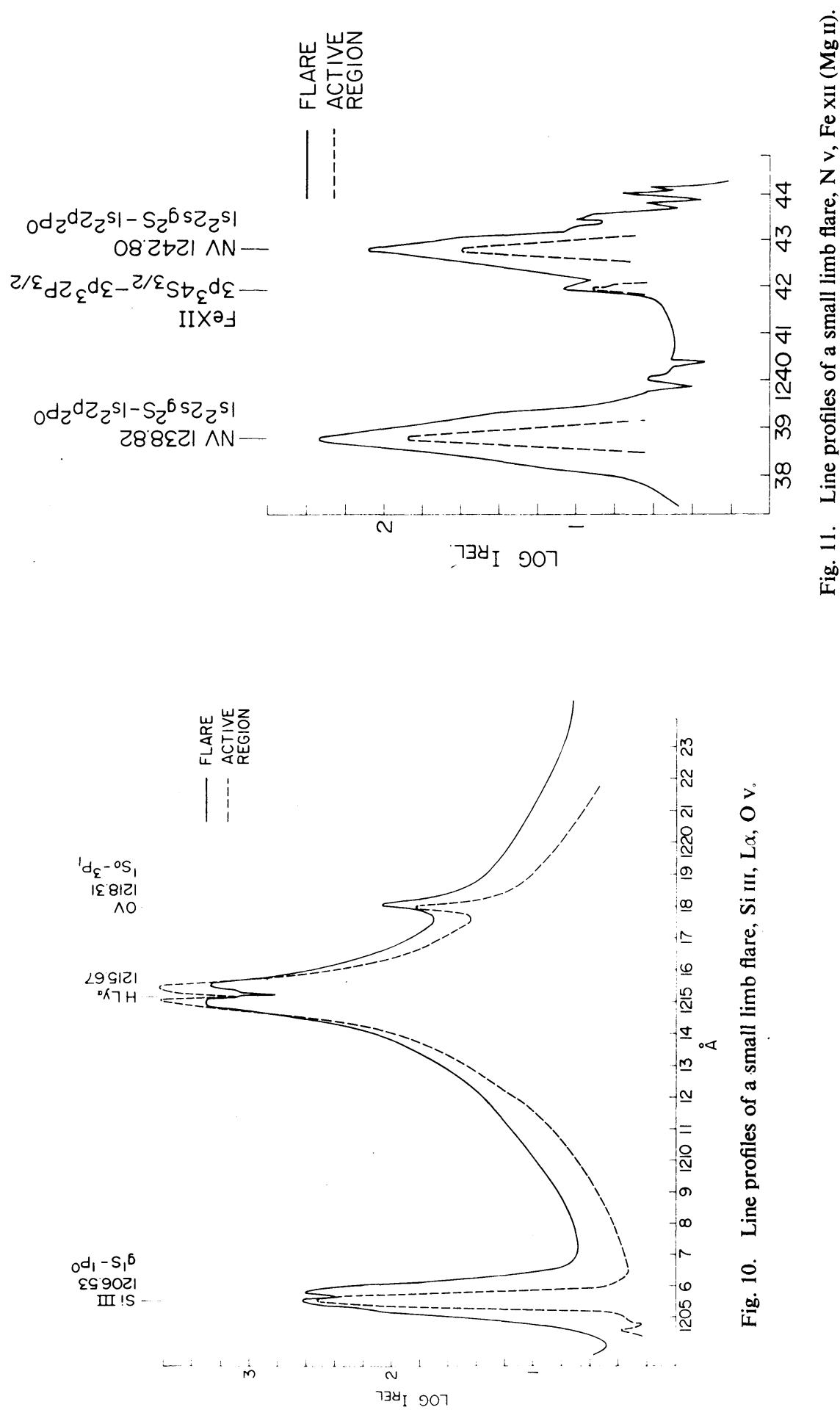
strong or stronger than the pure flare profile. An isolation of the flare profile can be done, if the latter shows a large shift.

In Figure 16 we demonstrate an example of strongly shifted $\mathrm{N} v$ lines. Assuming, that the blue wing of the composite profile is representative of the background profile,

TABLE I

Typical line-widths of optically thin lines in active regions and flares

\begin{tabular}{|c|c|c|c|c|c|c|}
\hline$\lambda$ & Ion & $T_{\mathrm{e}}$ & $\begin{array}{l}T_{\mathrm{K}} \times 10^{6} \mathrm{~K} \\
\text { active region }\end{array}$ & small flare & $\begin{array}{l}\xi_{0} \mathrm{~km} \mathrm{~s}^{-1} \\
\text { active region }\end{array}$ & small flare \\
\hline 1296 & Si III & $5 \times 10^{4}$ & 1.0 & 3.1 & 24 & 43 \\
\hline 1393 & Si IV & $7 \times 10^{4}$ & 1.1 & 8.4 & 26 & 70 \\
\hline 1402 & Si IV & $7 \times 10^{4}$ & 1.1 & 8.3 & 26 & 70 \\
\hline 1548 & C IV & $1 \times 10^{5}$ & 1.4 & 3.0 & 43 & 65 \\
\hline 1550 & C IV & $1 \times 10^{5}$ & 1.4 & 2.8 & 43 & 65 \\
\hline 1399 & O IV & $1.25 \times 10^{5}$ & 1.5 & 1.6 & 39 & 41 \\
\hline 1238 & $\mathrm{~N} v$ & $1.6 \times 10^{5}$ & 1.4 & 3.2 & 41 & 62 \\
\hline 1242 & $\mathrm{~N} v$ & $1.6 \times 10^{5}$ & 1.5 & 2.6 & 43 & 56 \\
\hline 1371 & $\mathrm{Ov}$ & $2.2 \times 10^{5}$ & 1.5 & 2.4 & 35 & 50 \\
\hline
\end{tabular}

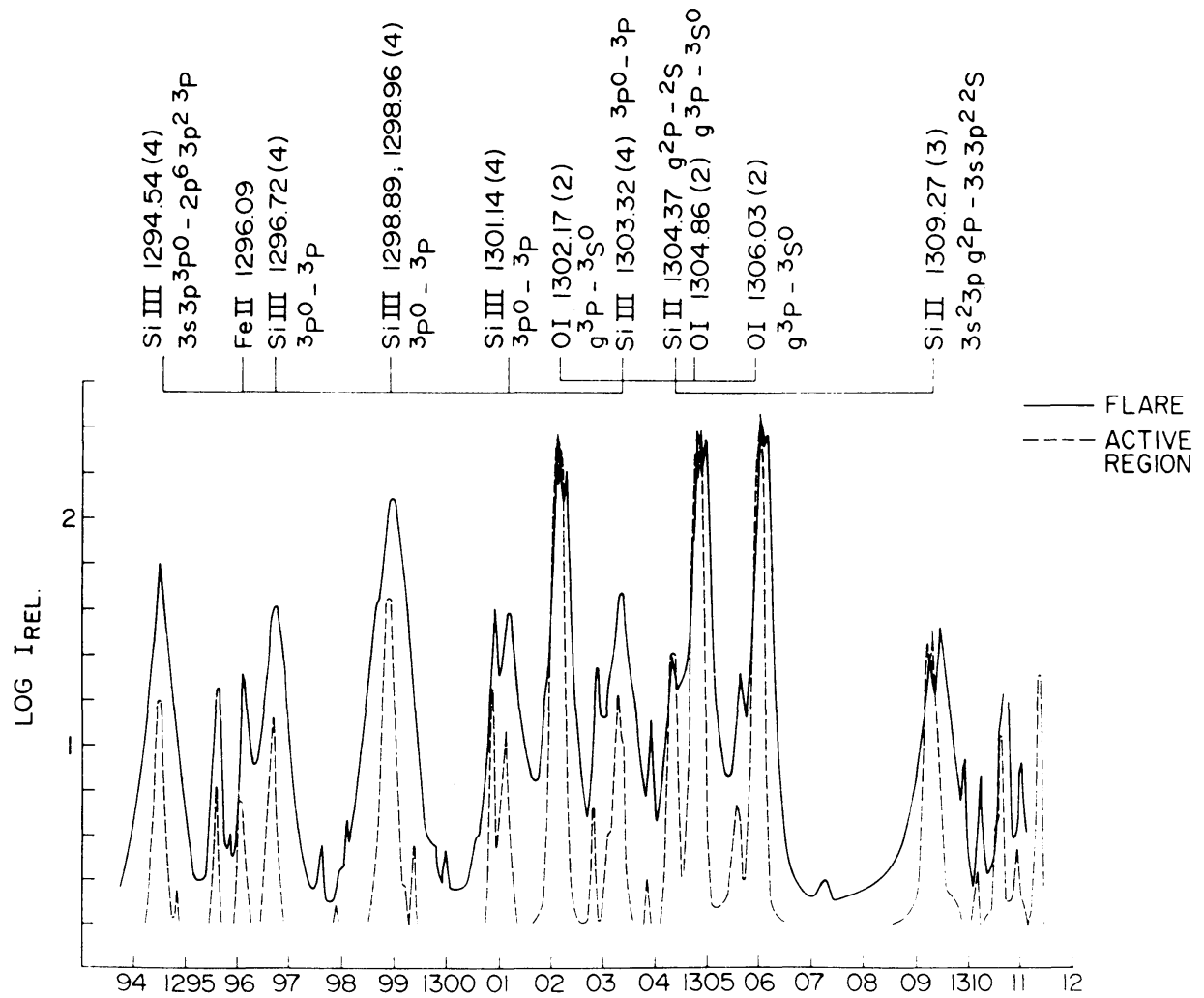

Fig. 12. Line profiles of a small limb flare, O I, Si II, Fe II, Si III. 
we determine the shift of the flare profile to be $68 \mathrm{~km} \mathrm{~s}^{-1}$ to the red and obtain for its halfwidth a Doppler velocity of $68 \mathrm{~km} \mathrm{~s}^{-1}$.

In Table I typical line width of optically thin lines in a flare spectrum compared with an active region are given. These lines are listed in order of increasing excitation temperature, covering a range from 50000 to $220000 \mathrm{~K}$. We notice the strong discrepancy between excitation and ion temperature, which exist already in the active region spectrum. (The same applies to the quiet Sun transition layer.) This discrepancy is greatly

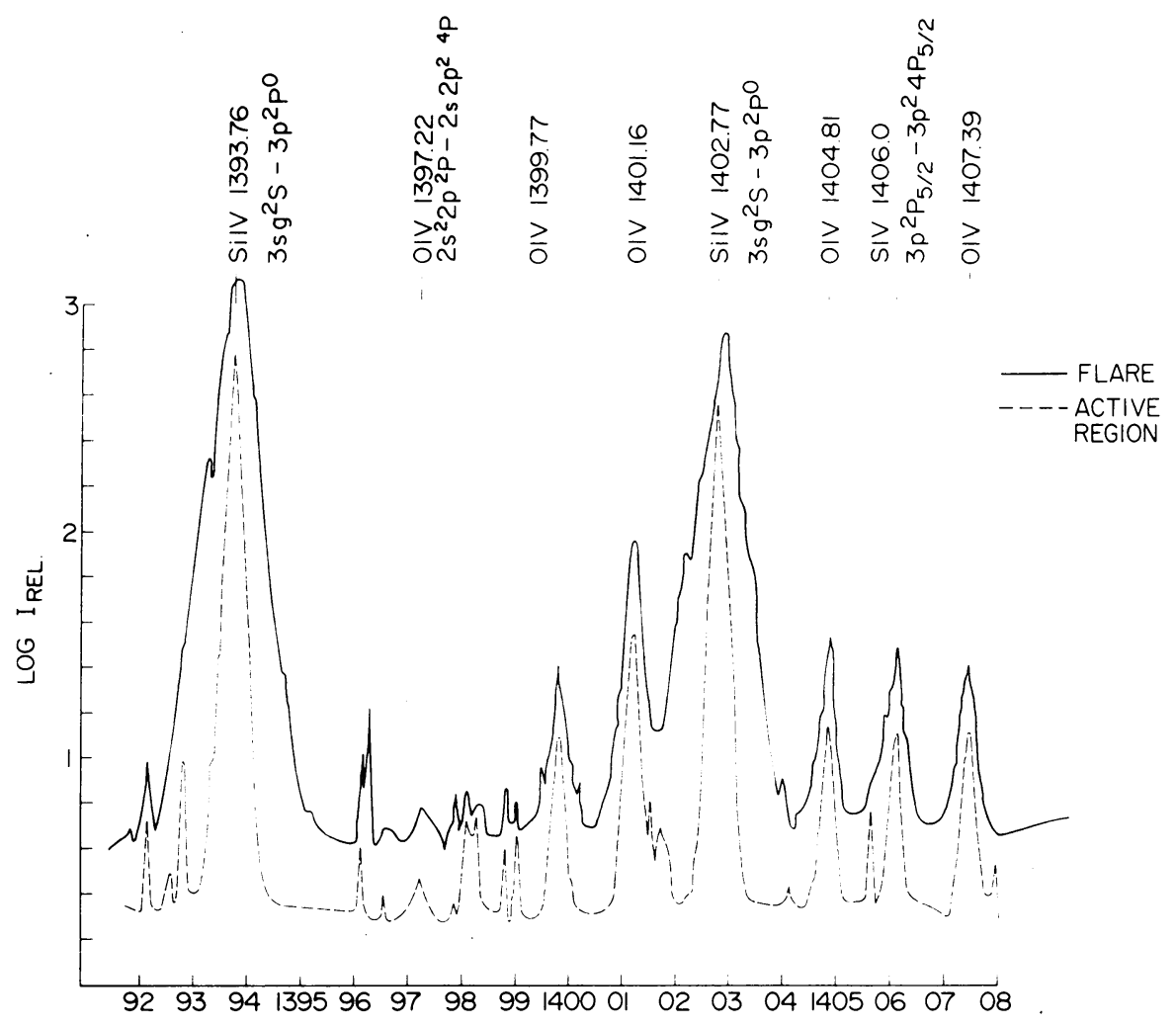

Fig. 13. Line profiles of a small limb flare, Si Iv, O Iv, S Iv.

enhanced in the flare spectrum, but we also note that the maximum ion temperature can be found at an excitation temperature of $70000 \mathrm{~K}$. At higher excitation temperatures, the ion temperatures decrease. The list shows that there is no change in the halfwidth of the $\mathrm{O}$ IV intersystem line indicating, that this line does not originate in the turbulent flare plasma. If we express the halfwidth of the lines in terms of Doppler velocities, we find $70 \mathrm{~km} \mathrm{~s}^{-1}$ as a maximum value for $\mathrm{Si}$ IV in this particular case. The term 'ion temperature' is used here in a very broad sense only as an expression for the measured halfwidth of the lines. It is perhaps not related to the temperature of the emitting gas, as will be pointed out below. 


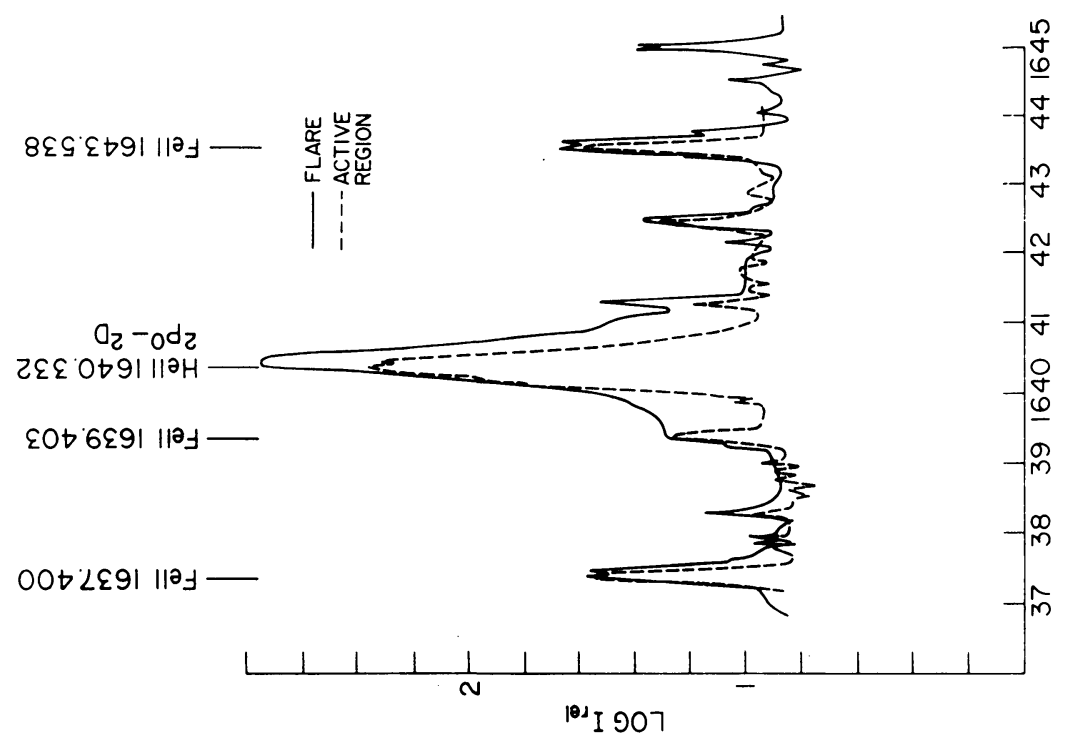

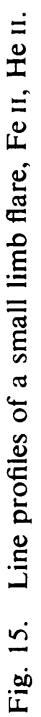

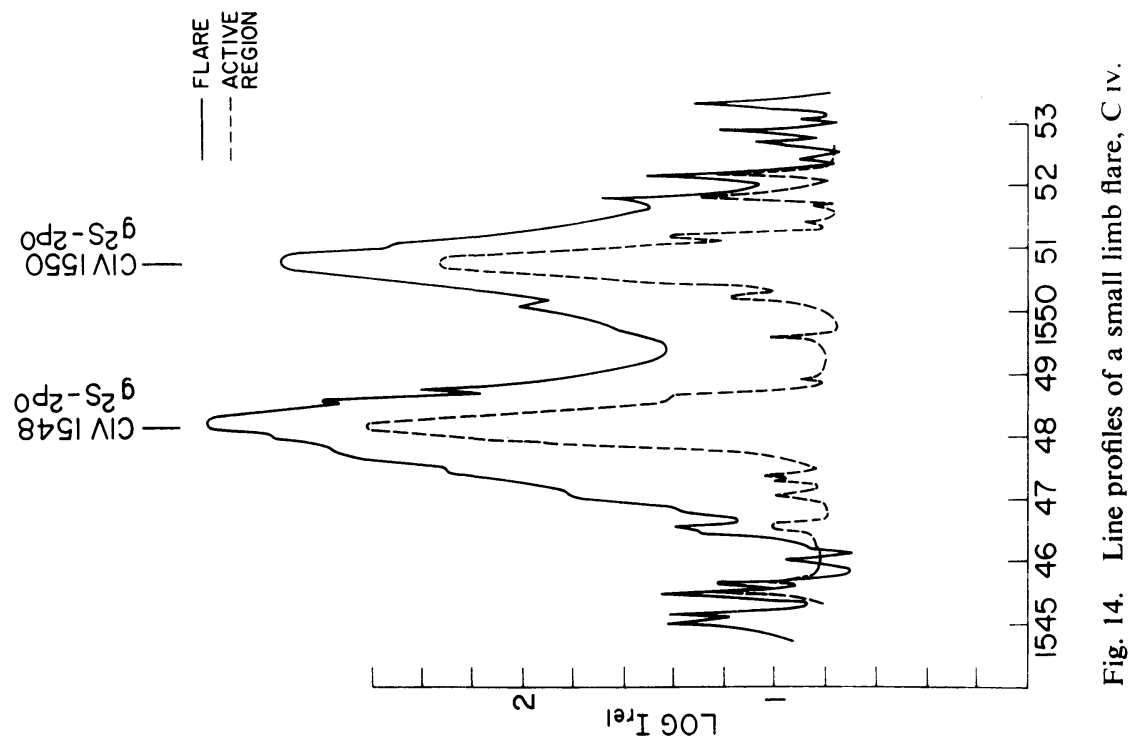


Starck broadening, microturbulence and macroturbulence are possible mechanisms which could be used to explain the very broad profiles of the transition zone lines in flares. In addition we must consider the possibility, that local strong electrical fields cause the high ion velocities. We believe, that the latter explanation is very unlikely, because if such fields would exist, the electrons should be accelerated to comparable energies like the ions and all atoms should be completely ionized. On the other hand, this possible mechanism should not be discounted completely until exact calculations disprove it. If such a mechanism would be working we would expect that the line width are proportional to the $z / m$ ratio of the specific ion. Such a dependence cannot be found. The $z / m$ ratio of C IV is twice as large as that of Si IV and yet the full width at half maximum of both lines is almost identical.

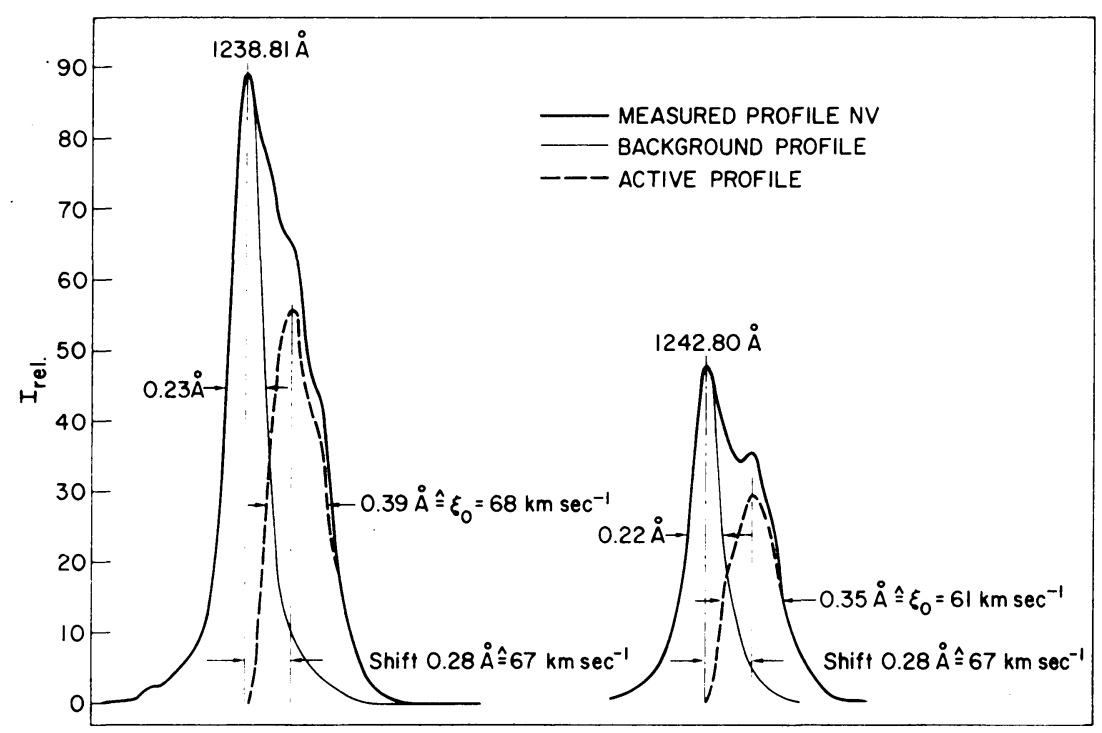

Fig. 16. Separation of $\mathrm{N} v$ flare line profiles from background line profiles.

Starck broadening by random electrons would yield to extreme high densities, larger than $10^{18} \mathrm{~cm}^{-3}$. Such densities can only be found in deep photospheric layers. A mechanism would have to be invented to ionize photospheric material locally to such high ion temperatures without the accompanying adiabatic expansion. If highly energetic electron beams can penetrate to such depth, than local ionization could take place. But calculations have shown, that even $100 \mathrm{KeV}$ electrons will only penetrate to layers, where the local density is approximately $10^{12} \mathrm{~cm}^{-3}$. These estimates have been done under the assumption, that the present models of the upper photosphere and chromosphere are valid, which is not necessarily the case.

Our observations do not show any correlation between line shifts and line broadening. Also, different ions can show different line shifts at the same time. Furthermore, the irregular structure of the line profiles along the slit (our spatial resolution is very 
marginal) indicate, that different areas of the flaring regions exhibit line shifts in different directions and of different magnitude. These fact seem to indicate, that local Doppler motions are causing the line broadening and the observed profiles are averaged over areas where these motions are not homogeneous. The size of these elements remains unknown. A mechanism still must be found, which is able to accelerate material to such high velocities without heating it up to very high temperatures. Sprays and surges are common features connected with solar flares. But a simple identification of our line broadening mechanism with sprays and surges, as they are observed in $\mathrm{H} \alpha$, cannot be made, because we observe blue shifts, red shifts and in many cases the lines are only broadened and not shifted.

However, if we explain the broadening of the transition zone lines by macroscopic Doppler effect, we must find an explanation for the fact, that the intersystem lines appear very strong at the very moment, when the turbulence, observed in the allowed transitions ceases. If the flare plasma as a whole exhibits an instability during the turbulent phase, an increased collision rate could de-excite the intersystem transitions. (This would be equivalent to an increased density during the turbulent phase.) If however, the line broadening mechanism is macroturbulence, then it is not easy to understand, why the sudden stop of the large scale movements should increase the intensity of the intersystem lines. It is therefore likely, that the broad lines of the allowed transitions reflect both, a strong turbulent motion within the flare plasma and local anisotropic acceleration of material. At the moment, when the Doppler broad-

TABLE II

Intersystem lines in flare spectrum

\begin{tabular}{|c|c|c|c|c|}
\hline$\lambda$ & Ion & Transition & $A\left(\mathrm{~s}^{-1}\right)$ & Remarks \\
\hline 1487 & N IV & $2 s^{2}{ }^{1} S_{0}-2 s 2 p^{3} P_{1}$ & $4.71(2)$ & enhanced after 'turbulent' phase \\
\hline 1218 & $\mathrm{Ov}$ & & $1.88(3)$ & no change during flare \\
\hline 1754.1 & $N_{\text {III }}$ & $2 s^{2} p^{2} P_{3 / 2} \cdots 2 s 2 p^{2}{ }^{4} P_{1 / 2}$ & $?$ & $\begin{array}{l}\text { very slightly weaker during } \\
\text { turbulent phase }\end{array}$ \\
\hline 1399.8 & $\mathrm{O}$ IV & $2 s^{2} p^{2} P_{1 / 2}-2 s p^{2}{ }^{4} P_{1 / 2}$ & $4.8(3)$ & enhanced after 'turbulent' phase \\
\hline 1401.1 & O IV & ${ }^{2} P_{3 / 2}-{ }^{4} P_{5 / 2}$ & $7.5(3)$ & enhanced after 'turbulent' phase \\
\hline 1404.8 & O IV & ${ }^{2} P_{3 / 2}-{ }^{4} P_{3 / 2}$ & $2.6(3)$ & enhanced after 'turbulent' phase \\
\hline 1407.4 & O IV & ${ }^{2} P_{3 / 2}-{ }^{4} P_{1 / 2}$ & $2.9(3)$ & enhanced after 'turbulent' phase \\
\hline 1660.8 & O III & $2 s^{2} 2 p^{2}{ }^{3} P_{1}-2 s 2 p^{35} S_{2}$ & $?$ & $\begin{array}{l}\text { slightly enhanced after 'turbulent' } \\
\text { phase }\end{array}$ \\
\hline 1666.1 & O III & ${ }^{3} P_{2}-{ }^{5} S_{2}$ & $?$ & $\begin{array}{l}\text { slightly enhanced after 'turbulent' } \\
\text { phase }\end{array}$ \\
\hline 1355.6 & $\mathrm{O}_{\mathrm{I}}$ & $2 s^{2} 2 p^{4}{ }^{3} P_{2}-2 s 2 p^{5}{ }^{5} S_{2}$ & $1.3(3)$ & enhanced during flare \\
\hline 1358.5 & $\mathrm{O}_{\mathrm{I}}$ & ${ }^{3} P_{1}-{ }^{5} S_{2}$ & $3.8(2)$ & enhanced during flare \\
\hline 1892.1 & Si III & $3 s^{2}{ }^{1} S_{0}-3 s 3 p{ }^{3} P_{1}$ & $8.3(4)$ & no change during flare \\
\hline 1204.3 & $\mathbf{S} \mathbf{v}$ & & $?$ & enhanced during early flare phase \\
\hline 1406.0 & S IV & $3 s^{2} 3 p^{2} P_{3 / 2}-3 s 3 p^{2}{ }^{4} P_{1 / 2}$ & $?$ & $\begin{array}{l}\text { slightly enhanced after 'turbulent' } \\
\text { phase }\end{array}$ \\
\hline 1416.9 & S IV & ${ }^{2} P_{3 / 2}{ }^{4} P_{3 / 2}$ & $?$ & $\begin{array}{l}\text { slightly enhanced after 'turbulent' } \\
\text { phase }\end{array}$ \\
\hline 1485.6 & $\mathbf{S}_{\text {I }}$ & $3 p^{43} P_{0}-3 p^{3} 3 p^{15} D_{1}$ & $2.3(6)$ & not identified \\
\hline
\end{tabular}


ening of the allowed transitions ceases not only the acceleration of material but also turbulent motion within the remaining flare plasma comes to a halt. It could also be, that the intersystem lines are emitted only as a recombination spectrum of the cooling corona above the explosive plage region. After the turbulence in the plage spectrum has ceased, no additional heating of the corona overlaying the plage takes place and cooling starts, which is reflected in the appearance of lines of cooler ions. The intersystem lines are all originating from relative 'cool' ions compared with the very hot ions (Fe XXI, Fe XxIV) emitting during the explosive phase of the flare.

Table II summarizes the observations of the intersystem lines in the 1973, June 15 flare. The list of lines has been compiled from Jordan (1973). Jordan's identifications and $A$-values have been listed together with remarks about the behavior of these lines in the flare spectrum.

In principle, one should be able, to derive lower limits for densities from the strength of these lines. However, the uncertainties are too large at the moment to give meaningful values. There is a wide margin of error in the atomic parameters. But worse, the collision rates in a highly turbulent plasma are a completely unknown quantity. For these reasons we rather refrain at the time being from trying to quote quantitative density values.

\section{Acknowledgements}

The spectra used for this analysis were taken by the Skylab Astronauts P. Conrad, Dr J. Kerwin, P. Weitz (Skylab I) and A. Bean, Dr O. Garriott, J. Lousma (Skylab II). I am indebted to K. Nicolas, who did the microphotometry of the limb flare spectrum and to Dr G. Doschek, O. K. Moe, K. Nicolas and Dr C. Moore Sitterly for helpful discussions. Dr R. Tousey is the Principal Investigator of the NRL Skylab Project. This work was supported by NASA DPR S-60404G.

\section{References}

Feldman, U., Doschek, G. A., Cowan, R. D., and Cohen, L.: 1975, Astrophys. J., in press. Jordan, C.: 1973, Nuclear Instr. Methods 110, 373.

Widing, K. G., 1975. This volume, p. 153. 